\title{
Efecto de la densidad de plantación y la poda en el Chile huacle en invernadero
}

\author{
Effect of planting density and pruning on the huacle Chili in the greenhouse \\ Gabino Alberto Martínez-Gutiérrez', Lucía Armin Langlé-Argüello', Miguel Urrestarazu², \\ Cirenio Escamirosa-Tinoco ${ }^{1}$, Martín Hernández-Tolentino ${ }^{1}$, Isidro Morales ${ }^{1 *}$
}

\begin{abstract}
RESUMEN
El chile huacle o chilhuacle es una variedad de Capsicum annum L. de importancia etnobotánica y cultural de Oaxaca, México. Se está produciendo una significativa reducción de su superficie cultivada al aire libre debido a diversos factores ambientales. El objetivo de este trabajo fue evaluar la producción y calidad en función del manejo cultural de la poda y la densidad de la plantación. Se realizaron dos experimentos simultáneos con pimiento huacle en Oaxaca (México), en un ciclo corto de cultivo de seis meses en un invernadero plástico sin control climático. El primero de densidad de plantación a 2, 3 y 4 plantas m² ${ }^{-2}$ y el segundo de poda a 2 , 4 tallos y sin poda. Se midieron algunos parámetros de crecimiento vegetativo, producción, de calidad del fruto y sólidos solubles totales. La mayor densidad de plantación incrementó significativamente el índice de área foliar, el rendimiento y producción de materia seca total de fruto, mientras que los parámetros de calidad de fruto no se vieron afectados. Las plantas podadas a 4 tallos no se afectaron en altura ni en grosor de tallo respecto a las sin poda. El índice de área foliar fue menor en la poda a dos brazos y no varió con la poda de 4 tallos y sin poda. El mayor rendimiento y producción de materia seca de fruto se obtuvo con las plantas a 4 tallos, mientras que la calidad de los frutos no se vio afectada.
\end{abstract}

Palabras clave: Capsicum annum L., chile huacle, chilhuacle, densidad de plantación, poda, planta tradicional

\begin{abstract}
The objective of this study was to evaluate the production and quality of pruning and planting density as a function of cultural management. Two simultaneous experiments with the huacle chili were conducted. Experiment 1 consisted of planting density treatments of 2, 3 and 4 plants $\mathrm{m}^{-2}$ with a principal pruning of 3 stems per plant. Experiment 2 consisted of a planting density of 3 plants $m^{-2}$ and three pruning treatments of 2 or 4 stems per plant and of no pruning. Growth, production and fruit quality was measured. The highest planting density significantly increased the leaf area index, yield and fruit production in terms of total dry matter. The fruit quality parameters were not affected. The leaf area index was lowest in the treatment with pruning to two branches and did not vary with pruning to 4 stems and no pruning. The highest yield and dry matter fruit production was obtained from plants with 4 stems, while the quality of the fruit was not affected.
\end{abstract}

Keywords: Capsicum annum L., huacle chili, chilhuacle, planting density, pruning, traditional plant

\section{Introducción}

La etnobotánica sin duda ocupa un lugar prominente en la ciencia mundial. Uno de los cultivos más importantes de la etnobotánica mexicana es el chile, debido a que forma parte de la cultura e identidad nacional. Los frutos de chile (Capsicum spp.) son esenciales en la alimentación (Ben-Chaim et al., 2006; De, 2003) y muchas veces son los frutos de cada región los que le dan la singularidad a la comida local. El chile huacle o chilhuacle es una variedad del género Capsicum propia del estado de Oaxaca, y se utiliza como ingrediente fundamental para preparar el mole negro. Tradicionalmente en el territorio oaxaqueño existen tres tipos de moles: negro, rojo y colorado. En la actualidad la elaboración del mole se ha extendido en gran parte del país. En

1 Instituto Politécnico Nacional, Centro Interdisciplinario de Investigación para el Desarrollo Integral Regional (CIIDIR-IPN) Unidad Oaxaca. Oaxaca. México.

2 Departamento de Agronomía, CIAIMBITAL, Universidad de Almería. Almería, España.

* Autor para correspondencia: imoralesg@ipn.mx 
el estado de Oaxaca, la región donde predomina originariamente el cultivo del chile huacle es la Cañada (Figura 1). Esta región se compone de dos áreas geográficas (Cuicatlán y Teotitlán), cuyos habitantes mayoritariamente son de los grupos étnicos cuicatecos, ixcatecos, mazatecos y nahuas (López-López, et al., 2016). El chile huacle ha sido catalogado como en peligro de desaparición, ya que en los últimos años su cultivo se ha restringido a pequeñas superficies que varían de 2500 a $5000 \mathrm{~m}^{2}$ (Agroproduce, 2005; López-López, et al., 2016). En el año 2015 sólo se cultivó un total de 10 ha a cielo abierto y mediante sistema de riego rodado con un rendimiento menor a una tonelada de chile deshidratado por hectárea (López-López et al., 2016). Esto se debe a los altos costos de producción, carencia de genotipos mejorados y la producción en otras regiones del país a menor costo pero con calidad y sabores distintos, lo que ha causado una reducción de su superficie sembrada a pesar de los elevados precios que alcanza en el mercado en relación con otros chiles.

La utilización de los sistemas de horticultura protegida puede ofrecer una oportunidad de manejo adecuado para incrementar la productividad en los ciclos tradicionales de cultivo. Algunos autores reportan que el uso de ambientes controlados, bajo un apropiado manejo agronómico, puede aumentar la producción y/o mejorar la calidad de los frutos en los cultivos de pimiento (Cebula, 1995).

En algunos invernaderos comerciales, el desarrollo del cultivo del pimiento está controlado por un patrón de poda a dos, tres o cuatro tallos principales (Jovicich et al., 2004; Urrestarazu, 2004), con el objetivo de concentrar la producción en las ramificaciones más vigorosas (Urrestarazu, 2004). $\mathrm{Al}$ evaluar la poda del pimiento bajo invernadero se han encontrado mayores rendimientos por planta y un aumento del número de frutos, así como un incremento en peso fresco de fruto al podar las plantas a cuatro tallos (Jovicich et al.,1999).

Una adecuada densidad de plantación puede llevar a la obtención de rendimientos óptimos. Sin embargo, una densidad muy alta o muy baja puede disminuir el rendimiento y el tamaño de fruto (Maboko et al., 2012; Urrestarazu, 2004). En ciertas regiones productoras de pimiento en horticultura protegida como España, la densidad de plantación en un cultivo de pimiento bajo invernadero es normalmente de 2 plantas $\mathrm{m}^{-2}$, aunque según el número de tallos por planta se puede variar la densidad hasta 3 plantas $\mathrm{m}^{-2}$ (Urrestarazu, 2004). Sin embargo, Dasgan y Abak (2003) obtuvieron un mayor rendimiento por unidad de superficie con 8 plantas $\mathrm{m}^{-2}$.

En esta variedad de chile no existe un programa de mejoramiento genético o un manejo

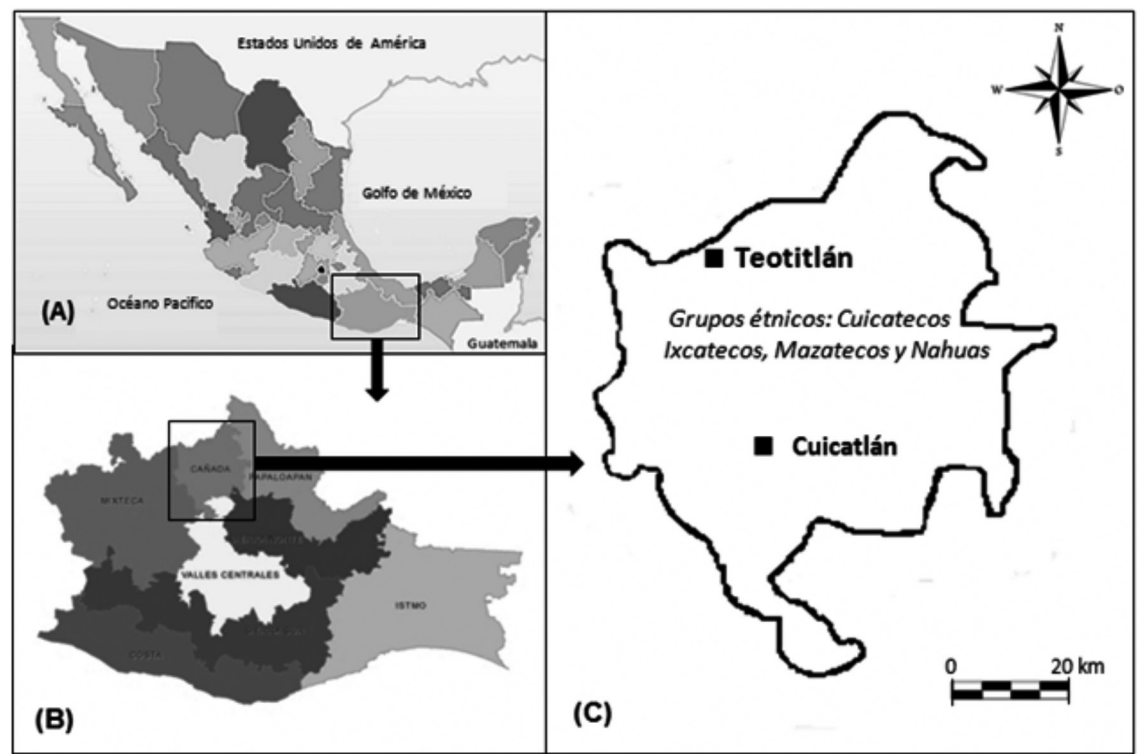

Figura 1. Mapa de ubicación de México (A), estado de Oaxaca (B) y la región de la Cañada (C), donde se encuentran los principales productores del chile huacle formados por grupos étnicos cuicatecos, ixcatecos, mazatecos y nahuas. 
cultural que determine el óptimo agronómico para aumentar la producción y/o mejorar la calidad de los frutos. Por consiguiente, al utilizar como referencia las prácticas realizadas en otra especie de Capsicum que cuenta con una gran superficie sembrada bajo invernadero a nivel mundial como lo es el pimiento, este trabajo pretende evaluar el manejo de la poda y densidad de plantación más adecuada para la producción de pimiento huacle en horticultura protegida.

\section{Materiales y métodos}

El estudio se llevó a cabo en el Centro Interdisciplinario de Investigación para el Desarrollo Integral Regional, Unidad Oaxaca (CIIDIR-IPNUnidad Oaxaca) del Instituto Politécnico Nacional (17 01 ' 31,45" de latitud norte, $96^{\circ} 43^{\prime} 12,07^{\prime}$ ' longitud oeste; altitud de $1526 \mathrm{msnm}$ ).

Las semillas se obtuvieron de un productor de la región de la Cañada. La siembra se realizó en bandejas de semillero de una densidad de 900 plantas por metro cuadrado, utilizando la mezcla Grow Mix (turba) y perlita a una proporción 3:1. 20 días después se plantaron en un invernadero plástico tipo túnel con ventilación cenital y lateral. Se usaron unidades de cultivo formadas por bolsas de polietileno de 17 litros, utilizando perlita como sustrato con un espaciamiento de $1.25 \mathrm{~m}$ entre hileras, y se ajustó el número de plantas de acuerdo a la densidad por tratamiento.

Para la nutrición de las plantas se utilizó un sistema de fertirriego con la solución recomendada por Urrestarazu (2004) para el cultivo sin suelo de pimiento sobre perlita. El trabajo se llevó a cabo mediante dos experimentos independientes. El experimento 1 consistió en los tratamientos de densidad de plantación a 2, 3 y 4 plantas $\mathrm{m}^{-2}$ con la poda principal de 3 tallos por planta. El experimento 2 incluyó tres tratamientos de podas a 2 a 4 tallos por planta y sin poda con un marco de plantación de 3 plantas $\mathrm{m}^{-2}$. Durante el ciclo de cultivo se tomaron las medidas de los datos de crecimiento vegetativo y producción total. La calidad de fruto se evaluó mediante un submuestreo con cinco frutos por repetición. Se midió la longitud, anchura de fruto y grosor de pericarpio. Los sólidos totales solubles ( ${ }^{\circ} \mathrm{Brix}$ ) se midieron por refractometría mediante un refractómetro de mano marca Atago PAL-1. La materia seca se obtuvo determinando el peso fresco de tres pimientos con una báscula analítica de centésima de gramo de precisión, una vez desecado en una estufa de aire forzado hasta obtener peso constante. El índice de área foliar (IAF) se estimó según Escalante y Kohashi (1993), mediante la ecuación: $\mathrm{IAF}=\mathrm{AF} * \mathrm{DP} 10,000 \mathrm{~cm}^{2}$, donde: IAF: Índice de área foliar; AF: Área foliar por planta $\left(\mathrm{cm}^{-2}\right)$; DP: Número de plantas $\mathrm{m}^{-2}$. Mientras que el área foliar se calculó utilizando el modelo propuesto por De Swart et al. (2004), para el método no destructivo de obtención de área foliar en Capsicum spp: $\mathrm{AF}=(0,61906 * \mathrm{~L} * \mathrm{~W})+\left(0,2060 * \mathrm{~W}^{\wedge} 2\right)-$ $(0,5142 * W)$, donde: AF: Área foliar; L: Largo de la hoja; W: Ancho de la hoja.

Para cada experimento se utilizó un diseño experimental completamente al azar, con un total de 3 tratamientos con 8 repeticiones. La unidad experimental constó de 3 plantas. Se realizó un análisis de la varianza y la correspondiente separación de medias. Se usó el programa Statgraphics Centurion ${ }^{\circledR}$ 16.1.15, 1999 para el tratamiento matemático de los datos.

\section{Resultados y discusión}

El principal uso del pimiento huacle es la elaboración del tradicional mole, de amplio consumo en todo el estado de Oaxaca. Por ello la producción que supondría un mayor beneficio económico podría ser aquella que genere una mayor biomasa en peso seco por superficie de producción.

\section{Efecto sobre el desarrollo vegetativo}

La densidad de la plantación afectó significativamente los parámetros de crecimiento de las plantas medidas (Tabla 1). El potencial fotosintético por una misma superficie foliar se incrementaba a favor del tratamiento con mayor densidad de plantas por unidad de superficie (en un 54 y 16\%, para la densidad de 2 a 3 y de 3 a 4, respectivamente). Castilla (1996) reporta que una adecuada densidad optimiza la intercepción de radiación y permite incrementar la fotosíntesis para, con posterioridad y mediante técnicas de cultivo apropiadas, derivar la distribución de asimilados al fruto.

En el experimento de la intensidad de la poda se mostraron también diferencias significativas en los parámetros del número de hojas y el IAF. El número de hojas se incrementó en un $45 \%$ y $32 \%$ al pasar de 2 a 4 y de 4 a no podar las plantas 
Tabla 1. Parámetros de crecimiento vegetativo del cultivo de pimiento huacle en función de la densidad de plantación (DP, planta $\mathrm{m}^{-2}$ ) y la poda (P) realizada por el número de tallos principales (2 o 4) o sin podar (SP).

\begin{tabular}{cccccc}
\hline Tratamiento & Altura $(\mathrm{cm})$ & Grosor de tallo $(\mathrm{mm})$ & Número de hojas planta $^{-1}$ & Índice de área foliar $^{\mathrm{N}} \mathrm{DP}$ \\
\hline & 2 & $66,1 \mathrm{a}$ & $9,9 \mathrm{a}$ & $56,4 \mathrm{a}$ & $1,1 \mathrm{c}$ \\
& 3 & $67,7 \mathrm{a}$ & $10,1 \mathrm{a}$ & $55,6 \mathrm{a}$ & $1,8 \mathrm{~b}$ \\
& 4 & $64,4 \mathrm{a}$ & $9,1 \mathrm{a}$ & $47,3 \mathrm{~b}$ & $2,1 \mathrm{a}$ \\
\hline \multirow{2}{*}{$\mathrm{P}$} & 2 & $71,8 \mathrm{a}$ & $9,6 \mathrm{a}$ & $35,6 \mathrm{c}$ & $1,4 \mathrm{~b}$ \\
& 4 & $66,5 \mathrm{ab}$ & $9,6 \mathrm{a}$ & $51,8 \mathrm{~b}$ & $2,3 \mathrm{a}$ \\
& $\mathrm{SP}$ & $63,2 \mathrm{~b}$ & $9,2 \mathrm{a}$ & $68,6 \mathrm{a}$ & $2,3 \mathrm{a}$ \\
\hline
\end{tabular}

Letras distintas entre columnas indican diferencias significativas, Tukey $5 \%$.

respectivamente. El IAF permaneció constante en las plantas a 4 y sin poda.

\section{Efecto sobre el rendimiento}

Experimento 1. La densidad de plantación afectó significativamente los parámetros de rendimiento (Tabla 2). Los valores medios de rendimiento son inferiores a los reportados para invernadero de tecnología baja y un ciclo de cultivo corto por Valera et al. (2014). Sin embargo, puede considerarse suficiente para su beneficio comercial, ya que se trata de una variedad de alto valor etnobotánico para la población autóctona.

A 4 plantas $\mathrm{m}^{-2}$ se obtiene el mayor número de frutos (en un 32 y $23 \%$ para la densidad de 2 a 3 y de 3 a 4 respectivamente). El rendimiento se incrementó (21 y $10 \%$ para la densidad de 2 a 3 y de 3 a 4 respectivamente para peso fresco, y 14 y $4 \%$ para biomasa seca de fruto). Dasgan y Abak (2003) y Maboko et al. (2012) indican que al aumentar el número de plantas por metro cuadrado, se contribuye a la producción de frutos extras con un menor tamaño, lo que lleva a un mayor rendimiento en dicha superficie. El peso medio de fruto disminuyó un $20 \%$ al incrementar la densidad a 4 plantas $\mathrm{m}^{-2}$. Diversos autores señalan que un aumento en la densidad de población causa disminución en el rendimiento por planta, pero incrementa el rendimiento por unidad de superficie (Cebula, 1995; Lorenzo y Castilla, 1995; Stoffella y Bryan, 1988). En el experimento 2, la poda a 4 tallos presentó el mayor rendimiento (incrementando un $39 \%$ al podar de 4 a 2 tallos y disminuyendo un $28 \%$ al pasar de 4 tallos a plantas sin poda) y la biomasa seca de fruto mostró un comportamiento similar (en un 28 y $46 \%$ respectivamente). Al disminuir el número de tallos se afecta negativamente el número de frutos y positivamente su peso medio. Sin embargo, para los objetivos del trabajo estas variables no tienen efecto significativo. Estos resultados son similares a los encontrados por Esiyok et al. (1994) y Muro et al. (1994) al realizar un estudio sobre el efecto de la reducción del área foliar en la disminución del rendimiento del pimiento, llegando a la conclusión de que la reducción de la cosecha era tanto mayor, cuanto

Tabla 2. Parámetros de rendimiento del cultivo de pimiento huacle en función de la densidad de plantación $\left(\mathrm{DP}\right.$, planta $\mathrm{m}^{-2}$ ) y la poda $(\mathrm{P})$ realizada por el número de tallos principales (2 o 4) o sin podar (SP).

\begin{tabular}{cccccc}
\hline Tratamiento & Rendimiento $\mathrm{g} \mathrm{m}^{-2}$ & Número de frutos $\mathrm{m}^{-2}$ & Peso fresco de fruto $^{-1}(\mathrm{~g})$ & Peso seco de fruto $\left(\mathrm{g} \mathrm{m}^{-2}\right)$ \\
\hline \multirow{2}{*}{$\mathrm{DP}$} & 2 & $957,2 \mathrm{c}$ & $29,2 \mathrm{c}$ & $32,78 \mathrm{a}$ & $71,12 \mathrm{c}$ \\
& 3 & $1160,6 \mathrm{~b}$ & $38,6 \mathrm{~b}$ & $30,07 \mathrm{ab}$ & $81,6 \mathrm{~b}$ \\
& 4 & $1281,6 \mathrm{a}$ & $47,4 \mathrm{a}$ & $27,04 \mathrm{~b}$ & $84,96 \mathrm{a}$ \\
\hline & 2 & $1196,2 \mathrm{~b}$ & $30,8 \mathrm{c}$ & $38,84 \mathrm{a}$ & $92,1 \mathrm{~b}$ \\
$\mathrm{P}$ & 4 & $1668,6 \mathrm{a}$ & $44,2 \mathrm{~b}$ & $37,75 \mathrm{a}$ & $118,48 \mathrm{a}$ \\
& $\mathrm{SP}$ & $1295,8 \mathrm{~b}$ & $57 \mathrm{a}$ & $22,73 \mathrm{~b}$ & $81,64 \mathrm{~b}$ \\
\hline
\end{tabular}

Letras distintas entre columnas indican diferencias significativas, Tukey $5 \%$. 
mayor era el nivel de defoliación. Los resultados fueron similares también a la tendencia que observan Dasgan y Abak (2003) de una mayor producción a 8 plantas $\mathrm{m}^{-2}$.

La densidad de plantación y la poda no tuvieron efectos significativos en los parámetros de calidad del fruto (Tabla 3). Frecuentemente en diversas hortalizas se reporta que no existe un importante efecto sobre los parámetros de calidad de los frutos tanto en cultivo de tomate (Morales y Urrestarazu, 2013) como en pimiento (Urrestarazu, 2004).

\section{Conclusiones}

El pimiento huacle puede introducirse en un sistema de cultivo intensivo bajo invernadero con rendimientos superiores a los obtenidos en los campos de la región. La densidad de plantación con 4 plantas $\mathrm{m}^{-2}$ tiene un mayor IAF, número de frutos, rendimiento en peso fresco y de materia seca total de fruto, lo que supone un mayor beneficio económico para el productor respecto a otras densidades de plantación más bajas. La poda a cuatro tallos tuvo el mejor rendimiento y mayor cantidad de materia seca sin afectar los parámetros de calidad.

\section{Agradecimiento}

Los autores agradecen al Instituto Politéncico Nacional, por el financiamiento otorgado al proyecto SIP20180457.

Tabla 3. Algunos parámetros de calidad de la producción del cultivo de pimiento huacle en función de la densidad de plantación (DP, planta $\mathrm{m}^{-2}$ ) y la poda (P) realizada por el número de tallos principales (2 o 4) o sin podar (SP).

\begin{tabular}{|c|c|c|c|c|c|c|}
\hline \multirow{2}{*}{\multicolumn{2}{|c|}{ Tratamiento }} & \multirow{2}{*}{$\frac{(\%)}{\text { Peso seco fruto }}$} & \multicolumn{3}{|c|}{$(\mathrm{mm})$} & \multirow{2}{*}{$\begin{array}{l}\text { Sólidos solubles totales } \\
\left.\text { ( }{ }^{\circ} \text { Brix }\right)\end{array}$} \\
\hline & & & Ancho de fruto & Largo de fruto & Grosor de pericarpio & \\
\hline \multirow{2}{*}{ DP } & 2 & $7,43 \mathrm{a}$ & 52,17 a & $61,73 \mathrm{a}$ & $1,97 \mathrm{a}$ & $11,86 \mathrm{a}$ \\
\hline & 3 & $7,03 \mathrm{ab}$ & 50,83 a & $66,43 \mathrm{a}$ & $1,93 \mathrm{a}$ & $11,90 \mathrm{a}$ \\
\hline \multirow{4}{*}{$\mathrm{P}$} & 4 & $6,63 \mathrm{~b}$ & 48,67 a & $64,27 \mathrm{a}$ & $1,97 \mathrm{a}$ & $12,21 \mathrm{a}$ \\
\hline & 2 & $7,70 \mathrm{a}$ & $50,80 \mathrm{a}$ & 66,65 a & $2,00 \mathrm{a}$ & $12,96 \mathrm{a}$ \\
\hline & 4 & $7,10 \mathrm{a}$ & $51,17 \mathrm{a}$ & $66,00 \mathrm{a}$ & $2,00 \mathrm{a}$ & $12,14 \mathrm{a}$ \\
\hline & SP & $6,30 \mathrm{~b}$ & $49,70 \mathrm{a}$ & $60,57 \mathrm{a}$ & $1,90 \mathrm{a}$ & $11,87 \mathrm{a}$ \\
\hline
\end{tabular}

Letras distintas entre columnas indican diferencias significativas, Tukey $5 \%$.

\section{Literatura Citada}

Agroproduce.

2005. Sistema-producto Chile. Fundación Produce. Oaxaca, México.

Ben-Chain, A.; Borovsky, Y.; Falise, M.; Mazourek, M.; Kang, B.; Paran, I.; Jahn, M.

2006. QTL analysis for capsaicinod content in Capsicum. Theo. Appl. Genetics, 113: 1481-1490.

Castilla, $\mathrm{N}$.

1996. Influencia de la radiación solar en invernadero sobre la calidad de la producción hortícola. En: Productividad y calidad de pimiento tipo lamuyo c.v. kalifa: respuesta a la fertilización potásica. Proyecto Fin de Carrera. Escuela Politécnica Superior. Almería, España.

Cebula, S.

1995. Optimization of plant and shoot spacing in greenhouse production of sweet pepper. Acta Horticulturae, 412: 321-328.

Daşgan, H.; Abak, Y.K.

2003. Effects of Plant Density and Number of Shoots on Yield and Fruit Characteristics of Peppers Grown in Glasshouses. Turkish Journal of Agriculture and Forestry 27: 29-35.
De Swart, E.A.M.; Groenwold, R.; Kanne, H.J.; Tam, P.S.;

Marcelis, L.F.M.; Voorrips, R.E.

2004. Non-destructive estimation of leaf area for different plant ages and accessions of Capsicum annuum L. The Journal of Horticultural Science \& Biotechnology, 79 (05): 764-770.

De, A.K

2003. Capsicum. The genus Capsicum. Taylor and Francis. London \& New York. 256 p.

Escalante, E.J.A.; Kohashi, S.J.

1993. El rendimiento y crecimiento del frijol. Manual para la toma de datos. Colegio de Postgraduados, Montecillo, México. 84 p.

Esiyok, D.; Ozzambak, E.; Eser, B.

1994. The effects of stem pruning on the yield and earliness of greenhouse peppers (Capsicum annum L. grossum cv. Kandil and 11B-14). Acta Horticulturae, 366: 293-300.

Jovicich, E.; Cantliffe, D.J.; Hochmuth, G.J.

1999. Plant density and shoot pruning on yield and quality of a summer greenhouse sweet pepper crop in North Central Florida. Proc. 28th Nat. Agric. Plastics Congress. 
American Society for Plasticulture. Tallahasse, Florida, USA, pp. 184-190.

Jovicich, E.; Cantliffe, D.J.; Stoffella, P.J.

2004. Fruit yield and quality of greenhouse-grown bell pepper as influenced by density, container and trellis system. HortTechnology, 14 (04): 507-513.

López-López, P.; Rodríguez-Hernández, R.; Bravo-Mosqueda, E. 2016. Impacto económico del chile huacle (Capsicum annuum L.) en el estado de Oaxaca. Revista Mexicana de Agronegocios, 38: 317-328.

Lorenzo, P., Castilla, N.

1995. Bell pepper yield response to plant density and radiation in unheated plastic greenhouse. Acta Horticulturae, 412: 330-334.

Maboko, M.M.; Du Plooy, C.P.; Chiloane, S.

2012. Effect of plant population, stem and flower pruning on hydroponically grown sweet pepper in a shadenet structure. African Journal of Agricultural Research, 7 (11): 1742-1748.
Morales, I.; Urrestarazu, M.

2013. Thermography Study of Moderate Electrical Conductivity and Nutrient Solution Distribution System Effects on Grafted Tomato Soilless Culture. HortScience, 48 (12): 1508-1512.

Muro, J.; Colum, R.; Claimon, S.

1994. Efectos de la reducción del área foliar sobre la producción del pimiento (Capsicum annum L.). Invest. Agr., 1-9.

Statistical Graphics Corp, Statgraphics Centurion. 1999. Statistical Graphics Corp., Rockville, MD.

Stoffella, P.J., Bryan, H.H.

1988. Plant population influences growth and yields of bell pepper. Journal American Society Horticultural Science, 113: $835-839$

Urrestarazu, M.

2004. Tratado de cultivo sin suelo. $3^{\text {a }}$ Ed Mundi-Prensa Libros. Madrid, España. 914 p.

Valera, M.D.L.; Belmonte, U.L.J.; Molina, A.F.D.; López, M.A. 2014. Los invernaderos de Almería. Análisis de su tecnología y rentabilidad. Ed. Cajamar, Caja Rural. Almería, España. 504 p. 